\title{
Epithelial plasticity is crucial for pancreatic cancer metastatic organotropism
}

\author{
Aleksandra Adamska, Marco Falasca \\ Metabolic Signalling Group, School of Pharmacy and Biomedical Sciences, Curtin Health Innovation Research Institute, Curtin University, Perth, \\ Western Australia, Australia \\ Correspondence to: Marco Falasca, PhD. Metabolic Signalling Group, School of Pharmacy and Biomedical Sciences, Curtin Health Innovation \\ Research Institute, Curtin University, Perth, Western Australia 6102, Australia. Email: marco.falasca@curtin.edu.au. \\ Provenance: This is an invited Editorial commissioned by Section Editor Dr. Clive R. Da Costa (Principal Laboratory Research Scientist, Adult Stem \\ Cell Laboratory, The Francis Crick Institute, London, UK). \\ Comment on: Reichert M, Bakir B, Moreira L, et al. Regulation of Epithelial Plasticity Determines Metastatic Organotropism in Pancreatic Cancer. \\ Dev Cell 2018;45:696-711.e8.
}

Submitted Sep 29, 2018. Accepted for publication Oct 07, 2018.

doi: 10.21037/atm.2018.10.16

View this article at: http://dx.doi.org/10.21037/atm.2018.10.16

Pancreatic ductal adenocarcinoma (PDAC) is one of the most lethal types of cancer with very poor patient outcomes. The limited efficacy of PDAC therapies is due to both late diagnosis and early development of metastases, giving patients few therapeutic options (1). The main sites of metastatic spread found in PDAC patients are the liver and lungs, with the latter, although less common, yielding significantly better prognosis for patients (40.3 vs. 29.9 months) (2). However, the molecular events controlling the development of metastasis in respective tissues have remained under-reported thus far. Understanding these processes and their potential manipulation, leading to the switch between different metastatic sites, could provide novel therapeutic opportunities for PDAC patients.

Cancer metastasis is a multistep process requiring intravasation, local invasion, creation of a metastatic niche and colonisation in distant organs (3). In order to accomplish this, cells must undergo a series of events that adapt their phenotype to a changing environment. The most well studied process is epithelial-to-mesenchymal transition (EMT), which allows cells to shift between an epithelial adherent state to a mesenchymal motile and invasive state, facilitating cell migration to distant sites (4). Primarily, EMT is regulated at the genetic level by transcription factors, which co-ordinately repress epithelial genes involved in the expression of adherens and tight junction components (e.g., E-cadherin). Concomitant with this is the activation of mesenchymal genes driving epithelial cell plasticity and dissemination to local or distant sites (5). However, how these genetic events might regulate metastatic organotropism has remained unclear thus far. A recent study by Reichert et al. addressed the phenomenon of epithelial plasticity on metastatic organotropism in pancreatic cancer (6). The authors investigated the potential mechanisms regulating PDAC epithelial plasticity and cell fate "decision" between colonization in the liver or lungs. Using a variety of metastatic mouse models of PDAC, the authors studied the effects of the manipulation of p120 Catenin (P120CTN) expression, a protein involved in E-Cadherin binding and stabilization (7) on metastatic organotropism in PDAC. Interestingly, they observed that the formation of liver or lung metastasis in PDAC depends on the functional form of P120CTN, with liver metastasis requiring intact or mono-allelic P120CTN, whereas biallelic deletion of the gene shifts the metastatic burden towards lungs (Figure 1). One allele of p120ctn is sufficient to stabilize E-Cadherin in liver metastasis where high membranous staining of both proteins was detected in mouse models and human metastatic liver tissues. However, lung metastases appear negative for both E-Cadherin and P120CTN, suggesting the epithelial character of metastatic cells, which might not be MET-capable. The results were confirmed by inoculation of PDAC cells bearing different P120CTN status into mouse liver or lungs. Formation 


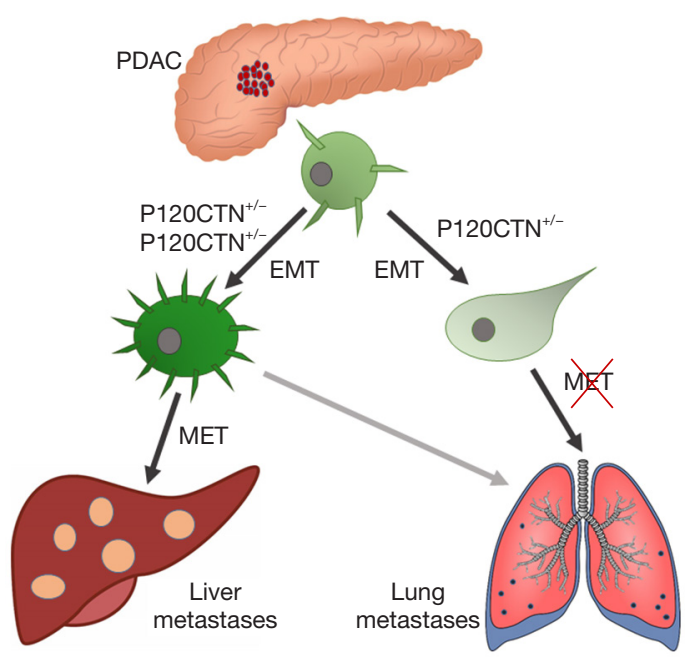

Figure 1 Regulation of membrane E-Cadherin by P120CTN status on epithelial plasticity affects metastatic organotropism in PDAC. PDAC, pancreatic ductal adenocarcinoma; EMT, epithelial-to-mesenchymal transition.

of liver metastasis was highly dependent on the active p120ctn form, whereas PDAC cells could colonize into lungs regardless of their p120ctn status, demonstrating that epithelial cell plasticity is not a determinant of cell metastatic potential. In addition, using a RNA-seq approach, the authors showed that loss of both alleles of p120ctn upregulate the pathways involved in EMT, which was not observed in the cells with mono-allelic or wildtype $p 120 c t n$. Taken together these data strongly support the hypothesis that cell epithelial plasticity under the control of P120CTN is a critical determinant of metastatic colonization site choice in pancreatic cancer. However, the reasons behind the demand of different organs for diverse cellular phenotypes are still to be explored. The differences between liver and lungs microenvironments were proposed as a potential factor contributing to the epithelial or mesenchymal preference of colonizing cells, nevertheless these aspects need further evaluation.

The understanding of the molecular mechanisms governing the development of metastasis in cancer is pivotal for the development of more effective therapies, especially in pancreatic cancer. Recent evidence suggests that the epithelial plasticity of cancer cells and the process of their dissemination involves a spectrum of diverse cell phenotypes and is far more complex than the direct transition between epithelial and mesenchymal states $(8,9)$. There are several theories on the molecular events governing the development of metastasis in cancer patients, including the aforementioned transcriptional regulation of protein expression, leading to the migration of single cells to the metastatic site. However recently, a more complex character of cell phenotypic plasticity has been shown, suggesting the existence of partial EMT (P-EMT), during which both epithelial and mesenchymal phenotypes may be exhibited by the cells, leading to internalization of proteins rather than their transcriptional repression (10). Collective cell migration is also observed in the P-EMT and considering the more epithelial phenotype of the clusters observed in previous studies and their higher metastatic colonization rates, the correlation between partial EMT and more aggressive tumours resulting in worse patient outcomes should be considered. Additionally, further exploration of cancer metastatic organotropism and development of tools enabling its genetic manipulation might present novel therapeutic opportunities, changing the perspectives and outlook for the better in PDAC patients.

\section{Acknowledgements}

M Falasca acknowledges support by Avner Pancreatic Cancer Foundation. A Adamska is supported by the Curtin University Health Sciences Faculty International Research Scholarships.

\section{Footnote}

Conflicts of Interest: The authors have no conflicts of interest to declare.

\section{References}

1. Adamska A, Domenichini A, Falasca M. Pancreatic Ductal Adenocarcinoma: Current and Evolving Therapies. Int J Mol Sci 2017;18:1338.

2. Yamashita K, Miyamoto A, Hama N, et al. Survival Impact of Pulmonary Metastasis as Recurrence of Pancreatic Ductal Adenocarcinoma. Dig Surg 2015;32:464-71.

3. Massague J, Batlle E, Gomis RR. Understanding the molecular mechanisms driving metastasis. Mol Oncol 2017;11:3-4.

4. Nieto MA, Huang RY, Jackson RA, et al. EMT: 2016. Cell 2016;166:21-45.

5. Ye X, Weinberg RA. Epithelial-Mesenchymal Plasticity: A Central Regulator of Cancer Progression. Trends Cell Biol 2015;25:675-86. 
6. Reichert M, Bakir B, Moreira L, et al. Regulation of Epithelial Plasticity Determines Metastatic Organotropism in Pancreatic Cancer. Dev Cell 2018;45:696-711.e8.

7. Ireton RC, Davis MA, van Hengel J, et al. A novel role for p120 catenin in E-cadherin function. J Cell Biol 2002;159:465-76.

8. Pastushenko I, Brisebarre A, Sifrim A, et al. Identification of the tumour transition states occurring during EMT. Nature 2018;556:463-8.

9. Li W, Kang Y. Probing the Fifty Shades of EMT in Metastasis. Trends Cancer 2016;2:65-7.

10. Aiello NM, Maddipati R, Norgard RJ, et al. EMT Subtype Influences Epithelial Plasticity and Mode of Cell Migration. Dev Cell 2018;45:681-95.e4.
Cite this article as: Adamska A, Falasca M. Epithelial plasticity is crucial for pancreatic cancer metastatic organotropism. Ann Transl Med 2018;6(Suppl 1):S53. doi: 10.21037/atm.2018.10.16 